\title{
VARIAÇÕES MORFOLÓGICAS EM GENÓTIPOS DE PINHEIRAS (Annona squamosa L.)
}

\author{
Taciana de Lima Salvador ${ }^{{ }^{*}}$; Eurico Eduardo Pinto de Lemos ${ }^{2}$; Tatiana de Lima Salvador ${ }^{1}$; Leila de Paula \\ Rezende ${ }^{2}$; Gilberto Dias Alves ${ }^{3}$.
}

\begin{abstract}
${ }^{1}$ Estudante de Pós-Graduação em Agronomia (PPGA), Centro de Ciências Agrárias (CECA), Universidade Federal de Alagoas (UFAL), BR 104 Norte, Km 85, Rio Largo, AL. CEP 57.000-100.

${ }^{2}$ Professor, Centro de Ciências Agrárias (CECA), Universidade Federal de Alagoas (UFAL), BR 104 Norte, Km 85, Rio Largo, AL. CEP 57.000-100.

${ }^{3}$ Professor, Institudo de Ciências Biológicas (ICB/UPE), R. Arnóbio Marquês, 310 - Santo Amaro, Recife - PE, CEP 50100-130.
\end{abstract}

*Autor para correspondência: Taciana de Lima Salvador; tacianasalvador@outlook.com

RESUMO: A caracterização morfológica através de descritores botânicos visíveis e mensuráveis é um dos métodos mais utilizados para distinguir cultivares de diversas espécies vegetais. Na família Anonnaceae, a caracterização morfológica de cultivares foi proposta apenas para a cherimóia (Annona cherimola Mill.) pela sua grande variabilidade natural e obtida através de programas de melhoramento em várias partes do mundo. A domesticação e a crescente importância de outros membros dessa família na produção de frutas tem feito surgir uma forte demanda por novas cultivares mais produtivas e com atributos comerciais desejáveis. No caso da pinheira (Annona squamosa L.), que há séculos vem sendo propagada sexuadamente, não existem cultivares registradas nos países produtores. Todavia, variações significativas tem sido observadas entre os tipos cultivados nas diversas regiões do mundo e também no Brasil. Algumas dessas plantas possuem grande apelo comercial e, se multiplicadas vegetativamente, poderiam dar origem a novas cultivares comerciais. Em Palmeira dos Índios, tradicional região produtora de pinha em Alagoas, atém do tipo Crioula predominante podem ser encontradas plantas com características fenotípicas bem diferentes. Este trabalho objetivou determinar descritores morfológicos mínimos para discriminar genótipos de pinheiras distintas do tipo padrão existente Crioula. Para tanto, se estudou 26 caracteres morfológicos típicos da cultivar padrão Crioula em 40 indivíduos escolhidos ao acaso em duas populações de pinheiras nos municípios de Palmeira dos Índios e Rio Largo, Alagoas. Os resultados de comparação dos 26 descritores morfológicos sugeridos apresentaram alto grau de similaridade entre as duas populações do tipo Crioula padrão, mas quando comparados com outros três genótipos apresentaram diferenças importantes capazes de os discriminar facilmente. Entre os descritores morfológicos mais discriminantes estão a cor, a textura e a cerosidade da casca do fruto, a cor dos ramos jovens, o formato e ondulação da lâmina foliar. Uma lista de descritores mínimos é proposta ao final.

PALAVRAS-CHAVE: annona, ata, fruta-do-conde, morfologia.

\section{MINIMUM MORPHOLOGICAL DESCRIPTORS TO DIFFERENTIATE SUGAR APPLE (Annona squamosa L.) GENOTYPES}

ABSTRACT: The morphological characterization through visible and measurable botanical descriptors is one of the methods used to distinguish different cultivars of plant species. In the Annonaceae family morphological characterization of cultivars has been proposed only for cherimoya (Annona cherimoya Mill.) because of its large genetic variability obtained through breeding programs worldwide. Domestication of other members of this family and their growing importance in fruit production has given rise to a strong demand for new cultivars with more productive and commercial attributes. In the case of sugar apple (Annona squamosa L.), which for centuries has been sexually propagated, there is no commercial cultivars established yet, although, significant morphological variations have been observed in the types grown in different regions of the world and also in Brazil. Some of these plants have great commercial appeal and, if multiplied vegetatively, could lead to new commercial cultivars. In Palmeira dos Indios, traditional producer of sugar apple region in Alagoas, beyond the typical genotype Creole can also be found plants with phenotypic characteristics very different. This study aimed to determine minimum morphological descriptors to discriminate genotypes of different sugar apple trees from the existing standard type 
Creole. Therefore, 26 morphological characteristics of the cultivar typical Creole have been studied in 40 randomly selected individuals in two different regions of Alagoas State: Palmeira dos Indios and Rio Largo. The results showed a high degree of similarity between the morphological descriptors chosen within the standard type of sugar apple in both populations, but when these descriptors were compared to other three genotypes significant differences were observed. Among the most discriminating morphological descriptors are color, texture and a waxy cover of the fruit and leaves, the color of the young branches, shape and waviness of the leaf blade. A list of minimum descriptors is proposed at the end.

KEYWORDS: Annona, ata, custard apple, morphology.

\section{INTRODUÇÃO}

A pinheira (Annona squamosa L.) é uma fruteira de importância econômica originária das terras baixas da América Central e México (Mosca et al., 2006). Acredita-se que a pinheira foi introduzida pela primeira vez no Brasil no início do século XVII pelo Conde Diogo de Miranda a partir de sementes trazidas da América Central (Pinto et al., 2005). Desde então essa espécie tem sido cultivada em quase todo o país, mas é no Nordeste que o seu cultivo tem se disseminado mais intensamente, apresentando mais de $90 \%$ de toda área cultivada do país (Lemos, 2014).

Por ter se dispersado no Brasil a partir de um conjunto inicial de sementes bastante restrito, a pinheira tem uma populaçãoaparentementehomogênea. Porém, considerando ser uma espécie preferencialmente alógama, por apresentar nas suas flores o fenômeno da dicogamia protogínica, as populações de pinheiras e de outras espécies de anonáceas nativas e compatíveis podem ter se entrecruzado naturalmente durante séculos, influenciando assim o surgimento de algumas variações fenotípicas e genotípicas interessantes (Lemos et al., 2010a).

Mutações também parecem ter ocorrido ao longo dos séculos e genótipos incomuns, tais como, frutos sem sementes, de coloração variando do verde ao roxo, exocarpo liso ou pontiagudo, plantas anãs ou desprovidas de cutícula serosa, distinções na sua textura, variações nas folhas, entre outros aspectos, podem ser observados entre as pinheiras cultivadas no Brasil. Partindo dessas diferenças, novas cultivares podem ser selecionadas, contribuindo assim para 0 crescimento da cultura no Brasil e no mundo (Lemos et al., 2010a).

O registro de cultivares é um processo importante para os programas de melhoramento, pois assegura a identidade genética e a qualidade varietal das cultivares, além dos direitos intelectuais dos obtentores. Adicionalmente ao registro, há a proteção das cultivares, a qual possibilita que órgãos e empresas públicas e privadas possam se beneficiar com a soma de recursos decorrentes dos direitos autorais obtidos, dando assim, sustentabilidade parcial ou total à continuidade dos programas de melhoramento (Carvalho et al., 2009).

A pinheira típica cultivada no Brasil e explorada comercialmente desde sua introdução possui características semelhantes que se repetem em milhões de plantas dando-lhe assim uma identidade morfológica facilmente reconhecível. Esse genótipo típico tem sido chamado comumente por produtores e pesquisadores de pinheira "Crioula".

Plantas com diferenças mensuráveis do tipo padrão Crioula têm sido observadas nas diferentes áreas de cultivo no Brasil e em outras partes do mundo. Em Alagoas, plantas com algumas características morfológicas claramente diferentes do tipo Crioula têm sido identificadas e vem despertando o interesse de produtores. Entre as variações que mais chamam a atenção estão a coloração do exocarpo podendo ser verde claro típico, verde intenso escuro, amarelado, avermelhado ou roxo; e a textura do exocarpo podendo este ser liso, rugoso ou pontiagudo. Todavia, observações mais atentas podem identificar outras variações que também possam ser discriminantes de genótipos diferentes.

A fixação de características genéticas de novos genótipos e a sua multiplicação em espécies de polinização cruzada como a pinheira somente é possível através de técnicas de propagação assexuada. Novos genótipos podem ser propagados vegetativamente por enxertia a partir de um tipo diferente morfologicamente caracterizado. Após multiplicados e agronomicamente caracterizados, tais tipos poderão então ser registrados como novas cultivares.

O International Plant Genetic Resources Institute (IPGRI) tem proposto descritores para muitas espécies vegetais cultivadas inclusive para a cherimóia (Annona 
cherimola Mill.) que, em sua maioria, caracteriza-se por uma lista simples de caracteres morfológicos que podem ser identificados com facilidade quando se compara genótipos distintos (Cherla, 2008). Variações na forma, tamanho, textura, cor entre outras observadas em troncos, ramos jovens, folhas, flores e frutos podem e devem ser usadas para separar cultivares de acordo com Serviço Nacional de Proteção a Cultivares (SNPC) (CARVALHO et al., 2009).

\section{MATERIAL E MÉTODOS}

Características morfológicas de 20 plantas de pinheiras típicas do genótipo Crioula com idade de doze anos foram avaliadas em um pomar comercial da Fazenda Paxiúba no município de Palmeira dos Índios, Alagoas ( $9^{\circ} 26 \square 22,4^{\prime \prime} \mathrm{S}, 36^{\circ} 41 \square 2,6^{\prime \prime}$ W e 295 $\mathrm{m}$ de altitude) e 20 plantas também do genótipo Crioula com seis anos de idade foram avaliadas no Centro de Ciências Agrárias no município de Rio Largo, Alagoas ( $9^{\circ} 27 \square 57^{\prime \prime} \mathrm{S}, 34^{\circ} 50 \square 1^{\prime \prime} \mathrm{W}$ e $127 \mathrm{~m}$ de altitude). Todas as plantas foram propagadas por sementes obtidas de frutos de várias origens e submetidas a tratos culturais semelhantes.

Os órgãos aéreos das plantas (ramos velhos, ramos jovens, folhas, flores, frutos e sementes) foram avaliados quanto a forma, o tamanho, a textura, a coloração e a presença ou ausência de estruturas associadas como cera e pelos. Pela natural dificuldade de observação, as raízes não foram consideradas no presente estudo.

\section{Caracterização morfológica}

Para 0 estabelecimento dos descritores mínimos utilizados na comparação dos indivíduos nas duas localidades utilizou-se como base a lista de descritores morfológicos proposta pelo International Plant Genetic Resources Institute (IPGRI) para cherimóia (Annona cherimola Mill.) (Cherla, 2008).

Amostras de 100 folhas, 100 flores, 10 frutos originários de polinização manual e 100 sementes de cada planta foram colhidas, colocadas em saco de papel e levadas ao Laboratório de Biotecnologia Vegetal do Centro de Ciências Agrárias da Universidade Federal de Alagoas em Rio Largo, AL, tomando-se o cuidado necessário para garantir a integridade das mesmas.

Para as características morfológicas dos frutos da pinheira foram considerados os aspectos formato, cor, textura, dimensões e presença de cera. Para as folhas, avaliações foram realizadas para o quesito cor da lâmina, cor das nervuras, formato, dimensões e presença de cera. Nas flores e nas sementes, as diferenças foram baseadas na coloração e dimensões das mesmas. As flores foram medidas a partir da inserção do pedúnculo no receptáculo floral até a ponta da pétala. Comprimento e largura foram realizados com o auxílio de um paquímetro.

Caracterização visual da morfologia de folhas e frutos foi realizada através da comparação da cor e textura dentro do mesmo grupo e entre grupo. A cor e a textura desses órgãos foram comparadas pondose amostras aleatórias de frutos das populações lado a lado por 20 vezes. Para se certificar de que esse era um bom critério para compor a lista de descritores mínimos, amostras de cada tipo foram examinadas separadamente para constatar se haviam divergências dentro de uma mesma população.

Para o caso de troncos e ramos, um exame e descrição individual foi feita para cada genótipo separadamente e, depois, os resultados quando consistentes foram comparados entreas duas populações.

Somente diferenças fáceis de serem observadas e simples de serem descritas por qualquer pessoa com um mínimo de treinamento e equipamentos foram consideradas para compor a lista de descritores mínimos proposta nesse trabalho.

\section{Testes de comparação entre genótipos diferentes utilizando os descritores mínimos}

Para testar a eficiência de 26 descritores mínimos propostos neste trabalho foram realizadas comparações morfológicas entre o genótipo padrão Crioula descrito e três outros acessos (genótipos) existentes na coleção de anonáceas do CECA-UFAL. Para tanto foram coletadas 10 ramos jovens (até $1 \mathrm{~cm}$ de diâmetro), 20 folhas maduras, 20 flores, 20 frutos jovens, 20 frutos maduros e 20 sementes dos acessos CECA 05, CECA 12 e CECA 21 e postos lado a lado para comparação com o padrão Crioula. Todas as partes avaliadas foram coletadas de duas plantas propagadas por enxertia de cada acesso existentes na coleção.

\section{RESULTADOS E DISCUSSÃO}

As comparações entre as duas populações de pinheira do genótipo Crioula de Palmeira dos Índios e Rio 
Largo, em Alagoas, apontaram semelhanças morfológicas suficientes que possibilitaram estabelecer um padrão de observações com consistência suficiente para se garantir que trata-se de tipos semelhantes e que, embora tenham origens distintas, apresentam os mesmos caracteres morfológicos propostos neste estudo.

A Tabela 1 propõe uma lista de 26 descritores mínimos os quais foram comparados entre as duas populações de pinheiras em campo. Para a maioria dos descritores estudados e apresentados na Tabela 1 as duas populações não apresentaram diferenças, exceto quando se utilizou caracteres quantitativos, tais como comprimento e largura de folhas, frutos e sementes. Mesmo assim, tais diferenças não parecem ser suficientes para caracterizar diferenças de genótipos e podem estar muito mais ligadas à condição ambiental a que cada população vivia no momento da avaliação (Palmeira dos Índios no Agreste e Rio Largo na Zona da Mata) do que propriamente a diferenças genéticas entre elas (Tabela 1).

Tabela 1. Lista de descritores morfológicos mínimos para a caracterização de dois genótipos de pinheira (Annona squamosa L.) em Alagoas, 2010.

\begin{tabular}{|c|c|c|c|}
\hline \multicolumn{2}{|r|}{ Descritores } & \multicolumn{2}{|c|}{ Populações do genótipo Crioula } \\
\hline & & Palmeira dos Índios & Rio Largo \\
\hline 1 & Cor tronco & Cinza claro & Cinza claro \\
\hline 2 & Cor ramo jovem & Verde claro opaco & Verde claro opaco \\
\hline 3 & Cor ramo adulto & Verde claro opaco & Verde claro opaco \\
\hline 4 & Cor da folha jovem & Verde claro opaco & Verde claro opaco \\
\hline 5 & Cor da folha adulta & Verde claro opaco & Verde claro opaco \\
\hline 6 & Formato folha & Elípitica & Elípitica \\
\hline 7 & Cor das nervuras & Verde claro & Verde claro \\
\hline 8 & Formato base da folha & Obtusa & Obtusa \\
\hline 9 & Limbo foliar & Lisa & Lisa \\
\hline 10 & Forma lamina foliar & Simétrica & Simétrica \\
\hline 11 & Cutícula (cera) & Presente & Presente \\
\hline 12 & Comprimento médio da lâmina foliar* & $12,5 \mathrm{~cm}$ & $12,3 \mathrm{~cm}$ \\
\hline 13 & Largura média da lâmina foliar* & $6,2 \mathrm{~cm}$ & $6,4 \mathrm{~cm}$ \\
\hline 14 & Cor flor & Creme amarelado & Creme amarelado \\
\hline 15 & Cor pétala interior & Creme & Creme \\
\hline 16 & Cor interna da base da pétala & Púrpura & Púrpura \\
\hline 17 & Comprimento da flor* & $2,4 \mathrm{~cm}$ & $2,6 \mathrm{~cm}$ \\
\hline 18 & Cor da casca do fruto & Verde claro/cinza & Verde claro/cinza \\
\hline 19 & Formato fruto & Cordiforme/redondo & Cordiforme/redondo \\
\hline 20 & Formato do gineceu & Arredondada & Arredondada \\
\hline 21 & Textura do exocarpo & Tuberculata com saliências suaves & Tuberculata com saliências suaves \\
\hline 22 & Comprimento médio do fruto** & $9,3 \mathrm{~cm}$ & $9,9 \mathrm{~cm}$ \\
\hline 23 & Largura média do fruto** & $9,0 \mathrm{~cm}$ & $10,1 \mathrm{~cm}$ \\
\hline 24 & Comprimento médio da semente ${ }^{*}$ & $1,2 \mathrm{~cm}$ & $1,4 \mathrm{~cm}$ \\
\hline 25 & Largura média da semente* & $0,6 \mathrm{~cm}$ & $0,5 \mathrm{~cm}$ \\
\hline 26 & Cor da semente & Marrom escuro & Marrom escuro \\
\hline
\end{tabular}


As avaliações preliminares em campo com três outros genótipos do Banco de Germoplasm de Pinheira do CECA-UFAL utilizando os mesmos 26 caracteres propostos mostraram que estes podem sofrer variações consideráveis (Tabela 2).

Tabela 2. Comparação utilizando descritores morfológicos mínimos para a caracterização de três genótipos de pinheira (Annona squamosa L.) em Alagoas, 2010.

\begin{tabular}{|c|c|c|c|c|c|}
\hline & \multirow{2}{*}{$\begin{array}{l}\text { Descritores } \\
\text { Mínimos }\end{array}$} & \multicolumn{4}{|c|}{ Comparação entre genótipos } \\
\hline & & Populações Crioula & $\begin{array}{c}\text { População } \\
\text { CECA } 05\end{array}$ & $\begin{array}{l}\text { População } \\
\text { CECA } 12\end{array}$ & $\begin{array}{l}\text { População } \\
\text { CECA } 21\end{array}$ \\
\hline 1 & Cor tronco & Cinza claro & Cinza claro & Cinza médio & Cinza escuro \\
\hline 2 & Cor ramo jovem & Verde claro opaco & Verde claro opaco & Verde escuro brilhante & Roxo escuro \\
\hline 3 & Cor ramo adulto & Verde claro opaco & Verde claro opaco & Verde escuro brilhante & Roxo opaco \\
\hline 4 & Cor da folha jovem & Verde claro opaco & Verde claro opaco & Verde escuro brilhante & Verde-arroxeada \\
\hline 5 & Cor da folha adulta & Verde claro opaco & Verde claro opaco & Verde intenso brilhante & Verde-arroxeada \\
\hline 6 & Formato folha & Elípitica & Elíptica & Elípitica & Elíptica \\
\hline 7 & Cor das Nervuras das folhas & Verde escuro & Verde claro & Verde escuro intenso & Roxo escuro \\
\hline 8 & Formato base da folha & Obtusa & Aguda & Obtusa & Aguda \\
\hline 9 & Limbo foliar & Lisa & Meio ondulado & Lisa & Ondulado \\
\hline 10 & Forma lamina foliar & Simétrica & Simétrica & Simétrica & Assimétrica \\
\hline 11 & Cutícula (cera) & Presente & Presente & Ausente & Presente \\
\hline 12 & Comprimento médio da lâmina foliar* & $12,5 \mathrm{~cm}$ & $12,7 \mathrm{~cm}$ & $13,6 \mathrm{~cm}$ & $10,4 \mathrm{~cm}$ \\
\hline 13 & Largura média da lâmina foliar* & $6,2 \mathrm{~cm}$ & $6,4 \mathrm{~cm}$ & $6,7 \mathrm{~cm}$ & $5,2 \mathrm{~cm}$ \\
\hline 14 & Cor flor & Creme amarelado & Creme amarelado & Creme amarelado & Roxo amarelado \\
\hline 15 & Cor pétala interior & Creme & Creme & Creme & Roxo claro \\
\hline 16 & Cor interna da base da pétala & Roxo claro & Roxo claro & Roxo claro & Roxo escuro \\
\hline 17 & Comprimento da flor* & $2,4 \mathrm{~cm}$ & $2,5 \mathrm{~cm}$ & $2,9 \mathrm{~cm}$ & $2,6 \mathrm{~cm}$ \\
\hline 18 & Cor da casca do fruto & Verde claro/cinza & Verde claro opaco & Verde intenso & Roxo intenso \\
\hline 19 & Formato fruto & Cordiforme/redondo & Cordiforme alongado & Cordiforme & Redondo \\
\hline 20 & Formato do gineceu & Arredondada & Cordiforme alongado & Cordiforme & Arredondado \\
\hline 21 & Textura do exocarpo & $\begin{array}{l}\text { Tuberculata com } \\
\text { saliências suaves }\end{array}$ & $\begin{array}{c}\text { Pontudo com saliências } \\
\text { angulosas }\end{array}$ & $\begin{array}{l}\text { Tuberculata com } \\
\text { saliências rugosas } \\
\text { acentuadas }\end{array}$ & $\begin{array}{l}\text { Tuberculata com } \\
\text { saliências suaves }\end{array}$ \\
\hline 22 & Comprimento médio do fruto** & $9,3 \mathrm{~cm}$ & $11,3 \mathrm{~cm}$ & $11,5 \mathrm{~cm}$ & $7,4 \mathrm{~cm}$ \\
\hline 23 & Largura média do fruto** & $9,0 \mathrm{~cm}$ & $6,5 \mathrm{~cm}$ & $9,4 \mathrm{~cm}$ & $7,6 \mathrm{~cm}$ \\
\hline 24 & Comprimento médio da semente* & $1,2 \mathrm{~cm}$ & $1,0 \mathrm{~cm}$ & $1,1 \mathrm{~cm}$ & $0,9 \mathrm{~cm}$ \\
\hline 25 & Largura média da semente* & $0,6 \mathrm{~cm}$ & $0,5 \mathrm{~cm}$ & $0,5 \mathrm{~cm}$ & $0,5 \mathrm{~cm}$ \\
\hline 26 & Cor da semente & Marrom escuro & Marrom escuro & Marrom escuro & Marrom escuro \\
\hline
\end{tabular}

*Média de 100 dados por planta; ${ }^{* *}$ Média de 10 dados por planta 
Os descritores morfológicos que mais discriminantes entre os genótipos Crioula, CECA 05, CECA 12 e CECA 21 avaliados foram a coloração de ramos jovens, folhas e casca dos frutos. Nestes casos observou-se que a coloração verde clara opaca das folhas deu lugar a um verde mais intenso e brilhante do genótipo CECA 12 ou com coloração roxa do genótipo CECA 21. Aparentemente, a coloração verde intenso e brilhante das folhas e frutos do genótipo CECA 12 estava associada a ausência de uma cobertura cerosa nesses órgãos. Por contraste, os outros genótipos Crioula e CECA 05 a presença de uma camada de cera estabelece um tom de verde claro fosco quase cinza. Essa camada de cera estabelece uma reação hidrofóbica na superfície das folhas, ramos jovens e frutos que a rigor não se molham com a aspersão de água sobre esses órgãos. 0 genótipo CECA 21 embora apresente a mesma camada cerosa das folhas e frutos sua coloração arroxeada é bastante distinta dos demais genótipos sendo facilmente separados dos demais.

Não foi possível avaliar se a ausência de cera sobre tais órgãos produzem vantagens ou desvantagens biológicas competitivas para a o genótipo CECA $12 \mathrm{em}$ relação aos demais. Todavia, a coloração verde intensa da casca dos frutos certamente produz um contraste que chama a atenção de produtores, comerciantes e consumidores e pode se constituir em um diferencial em favor dessa genótipo. Contudo, são necessários ainda mais estudos, principalmente sobre a sua resistência a pragas e doenças, conservação pós-colheita e aceitação do mercado consumidor antes que a mesma venha se tornar uma variedade comercial.

A cor da casca e da polpa dos frutos tem sido um foco importante em programa de melhoramento de frutas em todo o mundo. A atratividade da cor da casca dos frutos pode definir o sucesso ou o fracasso de uma nova variedade. De uma forma geral, cores mais intensas e brilhantes são preferidas em relação a casca mais claras e opacas (Pacheco et al., 2014). Em espécies que não possuem possibilidades de cores entre 0 amarelo intenso e o roxo, a cor verde mais intensa pode ser um diferencial importante para o sucesso comercial de uma cultivar (Souza, 2006; Santana et al., 2010).

$\mathrm{Na}$ ausência de variações organolépticas entre os quatro genótipos testados neste trabalho, a coloração dos frutos pode se constituir num importante diferencial de apelo comercial. Embora testes científicos para determinar a preferência dos consumidores não tenham sido conduzidos no mercado local, observações preliminares espontâneas apontam que a coloração verde mais intensa dos frutos do genótipo CECA 12 seriam mais atraentes do que o verde opaco dos genótipos Crioula, CECA 05 e roxo do CECA 21.

Estudos sobre características morfológicas de plantas tem como principal objetivo ampliar 0 conhecimento sobre determinada espécie, expandindo assim o reconhecimento e identificação das plantas de determinada região (Andrade et al., 2009). 0 estudo da morfologia do vegetal é de fundamental importância para caracterizar a diversidade de variedades que ocorrem na região estudada (Guimarães, 2005).

As avaliações feitas neste trabalho mostraram diferenças visíveis também na coloração das folhas dos genótipos de pinheiras avaliadas. 0 tipo Crioula apresenta uma coloração verde clara típica bem diferente dos genótipos CECA 21 (roxo) ou CECA 12 (verde intenso brilhante) fáceis de distiguir no meio de outras plantas de um mesmo pomar ou mesmo ainda no viveiro, pois tais variações acompanham a vida dos genótipos por toda a vida. Andrade e Martins (2007), em trabalho realizado com caramboleira (Averrhoa carambola L.), verificaram que é possível diferenciar as cultivares a partir das distinções morfológicas nas folhas, permitindo classificá-las mesmo em condições de viveiro.

A pinheira possui mecanismos morfológicos e fisiológicos que permitem conviver de forma produtiva em ambientes ecologicamente diferentes quanto à disponibilidade de água. A espécie pode produzir economicamente em zonas semi-áridas onde faz uso de mecanismos de resistência ao estresse hídrico para evitar a desidratação por transpiração e, na pinheira, a cutícula serosa em suas folhas participa ativamente deste mecanismo (Oliveira et al., 2005). O sucesso da cultura da pinha em zonas de baixa precipitação como o agreste de Alagoas e os sertões de Pernambuco e da Bahia mostra que essa é uma espécie com adaptações morfo-fisiológicas para estes ambientes.

A camada cerosa que recobre as folhas da pinheira é uma característica xeromórfica e tem como função reduzir a transpiração de água dos tecidos subepidérmicos (Lemos, 1994). Apesar da ausência da cutícula serosa nas folhas do genótipo CECA 12, esta falta parece não interferir no processo de crescimento e desenvolvimento da planta, assim como a formação 
das flores e dos frutos, pelo contrário, frutos dessa cultivar apresentam características de capacidade produtiva aparentemente superiores.

A textura encontrada nas cascas dos frutos das pinheiras do genótipo CECA 12 revela saliências mais acentuadas do que as demais utilizadas nesta comparação, sendo outra característica que pode ser usada para diferenciar as duas cultivares. Já o Genótipo CECA 05 apresentou exocarpo com textura bastante diferenciada com pontas agudas bem acentuadas lembrando a textura da variedade de atemóia Gefner.

As mutações espontâneas apresentamse como uma das fontes mais importantes para introdução de variabilidade genética em programas de melhoramento que visam obter cultivares de grande importância econômica, com maior produção, frutos mais firmes, de boa aparência, resistentes a doenças e saborosos (Soares Filho et al., 2003). Em tomateiro (Solanum lycopersicum L.) diversos mutantes têm sido identificados, onde apresentam aspectos alterados em sua coloração e processo de amadurecimento (Moura et al., 2004).

A comercialização no setor frutícola é dependente de apreciação positiva pelo consumidor que está relacionada principalmente com a aparência e as características sensoriais, além da garantia de segurança e qualidade, possibilitando o estabelecimento de estratégias diferenciadas de comercialização, e a orientação de programas de melhoramento genético de plantas frutíferas (Rombaldi et al., 2007).

Rombaldi et al. (2007) em pesquisa realizada com consumidores de frutas no Sul do Brasil, afirmaram que a aparência dos frutos constitui um dos fatores de maior relevância na decisão para adquirir uma determinada fruta. Notase que o consumidor é atraído pela aparência do fruto, e posteriormente, outras características são observadas como a coloração, ausência de lesões e podridões, aroma e sabor.

A lista de descritores mínimos pode ser usado para descrever as diferenças na morfologia de genótipos de pinheira e representa uma importante ferramenta que pode auxiliar melhoristas na caracterização de novas cultivares de pinheira.

Estudos relacionados às características pomológicas e agronômicas como produção/safra, peso do fruto, rendimento de polpa, brix, acidez titulável etc, poderão auxiliar na obtenção de dados completos referentes ao tipo padrão Crioula e outros genótipos que possam ser diferenciados pelos descritores minimos.

A avaliação de parâmetros fisiológicos decorrentes dessa variação (ausência de cera) no genótipo CECA12 sob diferentes condições de estresses ambientais poderá fornecer informações relevantes para predizer a sua resistência em campo.

A lista de descritores mínimos morfológicos aqui apresentada pode servir como padrão para 0 registro e proteção de cultivares de pinheira até que novos padrões genéticos de comparação possam ser propostos.

\section{AGRADECIMENTOS}

Os autores agradecem ao Conselho Nacional de Desenvolvimento Científico e Tecnológico - CNPq, e a Coordenação de Aperfeiçoamento de Pessoal de Nível Superior - CAPES pelo apoio financeiro.

\section{REFERÊNCIAS BIBLIOGRÁFICAS}

Andrade, R.A.A.; Lemos, E.G.M.; Martins, A.B.G.; Paula, R.C. Caracterização morfológica de plantas de rambutan. Acta Scientiarum Agronomy, 2009, 31, 4, 613-619.

Andrade, R.A.; Martins, A.B.G. Aspectos morfológicos de folhas na diferenciação de variedades de carambola. Comunicação Científica. Revista Brasileira de Fruticultura. 2007, 29, 2, 386-388.

Bioversity International and CHERLA. Descriptors for Cherimoya (Annona cherimola Mill.). Bioversity International, Rome, Italy; CHERLA Project, Malaga, Spain. 2008.

Carvalho, S.I.C.; Bianchetti, L.B.; Reifschneider, F.J.B. Registro e proteção de cultivares pelo setor público: a experiência do programa de melhoramento de Capsicum da Embrapa Hortaliças. Horticultura Brasileira, 2009, 27, 2, 135-138.

Guimarães, W.N.R. Caracterização morfológica e molecular de acessos de feijão-fava (Phaseolus 
Iunatus L., Fabaceae) da Coleção de Germoplasma do Departamento de Agronomia da UFRPE. 2005. 73p. Dissertação de mestrado em Melhoramento Genético de Plantas. Universidade Federal Rural de Pernambuco.

Lemos, E.E.P.; Barros, P.G.; Campos, R.S.; Salvador, T.L.; Santos, M.Q.C. Essential Descriptors for Sugar Apple (Annona squamosa L.) cultivars. In: 28th International Horticultural Congress, Lisboa. Book of Abstracts. Lisboa: International Society for Horticultural Science, 2010, 2, 175-175.

Lemos, E.E.P. Micropropagation, leaf abscission and sugar induced shoot regeneration in sugar apple (Annona squamosa L.) and soursop (Annona muricata L.). London: University of London, 1994. 207p. Thesis (PhD.). University of London.

Lemos, E.E.P. A produção de anonáceas no Brasil. Revista Brasileira de Fruticultura, 2014, 36, 77-85.

Mosca, J.L.; Cavalcante, C.E.B.; Dantas, T.M. Características botânicas das principais anonáceas e aspectos fisiológicos de maturação. Fortaleza: Embrapa Agroindústria Tropical, 2006.

Moura, M.A.; Galvão, H L.; Figeri, F.L. Crescimento e desenvolvimento de frutos do tomateiro "santa clara" e do seu mutante natural "firme". Ciência e Agrotecnologia, 2004, 28, 6, 1284-1290.

Oliveira, Z.P.; Queiroz, M.F.; Barros, P.G.; Campos, R.S.; Lemos, E.E.P.; Silva Neto, J.P. Recomendações técnicas para a cultura da pinha. 2005. Maceió:SEAGRIAL, 56p.
Pacheco, C.A.; Schinor, E.H.; Azevedo, F.A.; Bastianel, M.; Cristofani-Yaly, M. Caracterização de frutos do tangor TMXLP 290 para mercado de fruta fresca. Revista Brasileira de Fruticultura, 2014, 36, 4, 805-812.

Pinto, A.C.Q.; Cordeiro, M.C.R.; Andrade, S.R.M; Ferreira, F.H.; Filgueiras, H.A.C.; Alves, R.E.; Kimpara, D.J. Annona species. Fruits for the future, 5. 2005. International Centre for Underutilised Crops, University of Southampton, Southampto, UK. 263p.

Rombaldi, C.V.; Tibola, C.S.; Fachinello, J C.; Silva, J A. Percepção de consumidores do rio grande do sul em relação a quesitos de qualidade em frutas. Revista Brasileira de Fruticultura, 2007, 29, 3, 681-684.

Santana, F.A., Oliveira, L. A.; Viana, E. S.; Silveira, S. M.; Sousa, M. R.; Amorim, E. P. Avaliação da cor dos frutos de diferentes genótipos de bananeiras por colorimetro digital. In: Congresso Brasileiro de Fruticultura, 21., 2010, Natal. Anais... Natal: Sociedade Brasileira de Fruticultura, 2010. pdf 2378.

Soares Filho, W.S.; Sobrinho, A.P.C.; Passos, O.S.; Moitinho, E.D.B. "Maravilha": uma nova seleção de tangerina "Sunki $\square$. Revista Brasileira de Fruticultura, 2003, 25, 2, 268-271.

Souza, I V.B. Produção comercial de pinheira ( $A$. squamosa L.) em relação ao número de frutos por planta. 2006. 79p. Dissertação de Mestrado em Agronomia (Fitotecnia). Universidade Estadual do Sudoeste da Bahia. 